\title{
TRANSTORNO DEPRESSIVO EM PUÉRPERAS: AVALIAÇÃO DA PREVALÊNCIA E FATORES DE RISCO
}

Priscilla Santos Miguel, Lívia Calixto Batistela Novaes, Heliomar Pavao, Gilmara Rister e Alexandre Duarte Gigante

Universidade do Oeste Paulista - UNOESTE, Faculdade de Medicina, Presidente Prudente, SP.

\section{RESUMO}

A depressão é um transtorno muito prevalente em mulheres, sendo que o período gravídicopuerperal é de maior vulnerabilidade devido principalmente a alterações psicodinâmicas, fisiológicas e hormonais inerentes ao ciclo reprodutivo. Segundo alguns levantamentos, a prevalência de depressão pós parto varia entre 10 e $20 \%$, sendo que muitas mulheres não são diagnosticadas ou tratadas adequadamente em consultas de rotina. $\mathrm{O}$ rastreamento precoce dos sintomas de depressão pós parto é importante para o tratamento e garantia de um bom prognóstico, tanto para a mãe quanto para o bebê. Esta pesquisa teve o objetivo de estudar a prevalência de depressão em mulheres puérperas atendidas no ambulatório de puericultura do Hospital Regional de Presidente Prudente em até três meses de pós parto. Para isso, foi aplicada a escala de Edinburgh (EPDS), juntamente com um questionário sócio demográfico desenvolvido pelos autores com a finalidade de investigar os fatores de risco associados a essa patologia.

Palavras chave: Puerpério. Depressão. Escala de Edinburgh.

\section{PUERPERAL DEPRESSIVE DISORDER: PREVALENCE AND RISK FACTOR EVALUATION}

\begin{abstract}
Depression is a highly prevalent disorder in women, and the pregnancy-puerperal period is greater vulnerability due mainly psychodynamic, physiological and hormonal changes inherent in the reproductive cycle. According to some surveys, the prevalence of postpartum depression varies between 10 and $20 \%$, and many women are not diagnosed or treated properly during regularly scheduled appointments. The early screening of postpartum depression symptoms is important for the treatment and guarantee of a good outcome for both the mother and the baby. This research aims to study the prevalence of depression in women attending mothers in Presidente Prudente Regional Hospital childcare clinic within three months after childbirth. For this, the Edinburgh scale (EPDS) is applied along with a demographic questionnaire developed by the authors in order to investigate the risk factors associated with this disease.
\end{abstract}

Keywords: Postpartum depression. Edinburgh scale 


\section{INTRODUÇÃO}

O transtorno depressivo é, dentre os transtornos psiquiátricos, o mais comum entre mulheres, chegando a ser duas vezes mais prevalente em relação aos homens ${ }^{1}$. A prevalência do transtorno depressivo pós parto varia entre 10 e $20 \%$, mas apenas $18 \%$ das mulheres diagnosticadas são tratadas ${ }^{1}$. Esses números são aquém do ideal, pois alguns sintomas podem ser confundidos com a gravidez normal. Além disso, existe o fator cultural, a dificuldade da aceitação do quadro depressivo em um período que deveria ser de total felicidade ${ }^{1}$. As mulheres no puerpério são assistidas por diversos profissionais de saúde. Porém, estes profissionais focam seus atendimentos na saúde física, da mãe e do bebê ${ }^{2}$ e aspectos da saúde mental muitas vezes não são reconhecidos e quando são não são tratados de maneira adequada ${ }^{2}$. O rastreamento precoce desses transtornos é vital para o tratamento e para um bom prognóstico ${ }^{1}$. Destaca-se o início entre duas semanas a até três meses após o parto. Há humor depressivo, choro fácil, irritabilidade, perda de interesses pelas atividades habituais, sentimento de culpa, concentração prejudicada e pensamentos de morte $e^{2,3}$. Quanto mais graves e duradouros os sintomas, maior será a deterioração da relação mãe-bebê e repercussões negativas no desenvolvimento da criança ${ }^{3}$. Estudos recentes mostram a utilidade da aplicação de escalas de auto avaliação para triagem de mulheres com depressão em serviços de atendimento primário ${ }^{2}$. 0 instrumento mais utilizado hoje em dia para a detecção precoce de casos de depressão em mulheres grávidas e puérperas é a escala EPDS (Edinburgh Postpartum Depression Scale) ${ }^{1}$. Esta escala foi desenvolvida e testada em diversos países apresentando elevada sensibilidade e especificidade ( $86 \%$ e $78 \%$ ) na detecção de pacientes com depressão pós parto. Tem sido investigados inúmeros fatores de risco para a depressão pós parto. Através de uma meta-análise de 84 estudos, Beck ${ }^{7}$ identificou 13 fatores de risco: depressão pré-natal, baixa autoestima, estresse relacionado com os cuidados infantis, ansiedade pré-natal, estresse associado com condições de vida adversas, baixo suporte social, relação marital complicada, história pessoal de depressão, dificuldades temperamentais do bebê, melancolia pós-parto, estado civil, condição socioeconômica e gravidez desejada/não desejada. 0 objetivo desse artigo é evidenciar a elevada prevalência e impacto negativo para a puérpera, sua família e o bebê, sendo necessário que se dê atenção ao diagnóstico precoce. Trazendo à tona esta problemática pode-se prestar melhor atendimento a estas pacientes.

\section{METODOLOGIA}

Foi realizado um estudo de campo, transversal, com abordagem qualitativa e quantitativa, sendo a amostra composta por $53(n=53)$ pacientes puérperas em seguimento médico no Ambulatório de Puericultura do Hospital Regional de Presidente Prudente (HRPP). Para caracterização da amostra foi aplicada a escala EPDS (Edinburgh Postpartum Depression Scale) ${ }^{8}$ validada para o português pelo estudo de Santos e colaboradores em $2000^{6}$ e um questionário desenvolvido pelos autores, com dados sócio demográficos (Anexo 2). A escala foi aplicada no ambulatório com pacientes durante uma consulta de rotina do serviço em entrevista individual e teve duração média de 10 minutos. Foram incluídas na pesquisa as pacientes com idade de 18 a 35 anos, puérperas, até três meses após o parto. Todas as participantes assinaram Termo de Consentimento Livre e Esclarecido. Os dados foram obtidos pela aplicação da escala EPDS constituída por 10 itens, com quatro alternativas fechadas convertidas em pontos $(0,1,2$ e 3 pontos), de acordo com a gravidade crescente dos sintomas, sendo as questões 3, 5, 6, 7, 8, 9 e 10 foram cotadas inversamente $(3,2,1,0)$. Cada item é somado aos restantes para obter a pontuação total. Uma pontuação de 12 ou mais indica a probabilidade de depressão, mas não a sua gravidade. A EPDS foi desenhada para complementar, e não para substituir a avaliação clínica. Para análise estatística foi utilizado o Statistical Package for Social Sciences (SPSS). Foi realizada a estatística descritiva para as questões, sendo utilizado o teste qui-quadrado para comparações ou teste de Fisher quando apropriado. Um $\mathrm{p}<0,05$ será considerado estatisticamente significativo. 
Esse artido é aprovado pelo Comitê de Ética em Pesquisa com o numero 2389. Sendo que o numero da CAAE é 41125415.0.0000.5515.

\section{RESULTADOS}

Os resultados obtidos na análise das 53 pacientes $(n=53)$ podem ser vistos a seguir. A priori, observamos que a média de idade das pacientes consideradas deprimidas foi de 29,67 anos, com uma variação de 5,53 pontos para mais ou para menos. A média de idade do grupo de pacientes não deprimidas foi de 25,76 anos, com uma variação de 4,63 pontos para mais ou para menos. Sendo portanto significante estatisticamente a maior média de idade entre as pacientes puérperas deprimidas. A posteriori, em relação ao estado civil, escolaridade, renda e trabalho atual das pacientes estudadas, observamos que não houve diferença estatística significativa nestes itens. Com relação ao número de filhos como representado no gráfico 1 a seguir, foi visto que o grupo de pacientes deprimidas, tinham em média 2,42 filhos, com variação de 1,00 filho para mais ou para menos e as pacientes não deprimidas em média tinham 1,68 filhos, com variação de 0,72. Observamos ainda que complicações com o bebê e necessidade de internações do mesmo aumentam de forma significativa a chances de quadros depressivos na mãe. Encontramos $66.7 \%$ de mães deprimidas contra $34,1 \%$ das não deprimidas em caso de internação do bebê e $66,7 \%$ contra $29,3 \%$ em caso de complicações com o bebê. Em contrapartida complicações da mãe não foram estatisticamente significativa na determinação de quadro depressivo. Com relação as mães que tiveram ajuda para cuidar do bebê a maioria delas, 35 pacientes $(85,4 \%)$ não apresentaram quadro depressivo no puerpério. Quanto aos itens pesquisados: história de transtornos psiquiátricos prévios, história psiquiátrica na família, e problemas conjugais, não observou diferença estatisticamente significativa nesses itens.

\section{DISCUSSÃO}

A prevalência de Depressão pós parto (DPP) foi de $29,27 \%$, preconiza-se que possíveis variações para mais ou menos podem estar relacionadas ao método de diagnóstico, características sócio demográficas dos sujeitos, bem como ao período do puerpério em que a mulher foi avaliada e inter-regionais. Alguns estudos têm sua abordagem nos três primeiros trimestres pós-parto ${ }^{4}$, enquanto outros abordam a partir da $12^{a}$ semana pós-parto ${ }^{5}$. Outras fontes defendem seu aparecimento, na maioria dos casos, nas primeiras quatro semanas após o parto ${ }^{11}$, alcançando sua intensidade máxima nos seis primeiros meses ${ }^{5}$. Além disso, a prevalência encontrada acima da descrita na literatura parece indicar que, em muitas mulheres entrevistadas, podem ter sido diagnosticadas como possível depressão pós parto mas na verdade estavam vivendo um período conhecido como blues puerperal, quando a mãe vivencia sintomas próximos de depressão porém de forma mais branda e em um período curto. Quanto aos fatores de risco: gestação não planejada, pouca idade materna, baixo nível sócio econômico, grande número de filhos, o fato da mãe não estar casada, problemas no relacionamento conjugal, ajuda insatisfatória nos cuidados com o filho, desemprego, baixo peso ao nascer, doenças psiquiátricas anteriores ou durante a gestação $^{2,12}$. O item "ajuda com o bebê" foi bastante interessante, uma vez que nos trouxe resultados significativos quanto a importância de algum tipo de ajuda para os cuidados com a criança. Dessa forma foi visto nesse estudo que a grande maioria das pacientes que tiveram ajuda para cuidar do bebê não apresentaram episódio depressivo no pós parto. A presença de transtornos psiquiátricos anteriores ou mesmo durante a gestação é predisponente para quadro depressivo. No entanto o estudo deste fator de risco não encontrou diferença estatisticamente significativa neste aspecto. Além disso, quanto ao fator de presença de transtornos psiquiátricos na família a nossa pesquisa também não apontou o registro de casos depressivos para este fator de risco com significância estatística. Estes achados podem ser decorrentes do presente estudo ter 
abordado um número pequeno de pacientes $(n=53)$, ou mesmo devido à falta de conhecimento das mesmas a respeito do que seria considerado como distúrbio psiquiátrico.

\section{CONCLUSÃO}

Nem sempre é fácil para o obstetra identificar o diagnóstico de DPP. Por vezes os sintomas podem ser confundidos com o blues puerperal, condição normal onde ocorrem os ajustes emocionais relativos ao ganho de uma criança. As pacientes diagnosticadas com DPP foram devidamente encaminhadas para o Ambulatório de Psiquiatria do Hospital Regional de Presidente Prudente para tratamento adequado. Diversos achados neste estudo corrobora com a literatura, ratificando a importância da EPDS como instrumento adequado de triagem da depressão pósparto, podendo ser inclusive implementada na rede pública de saúde devido a sua facilidade, rapidez de aplicação, baixo custo e possibilidade de aplicação por qualquer profissional de saúde. Concluímos que o amplo uso da escala pode ser associado a um aumento nos índices de diagnóstico e tratamento da doença, minimizando assim seus possíveis efeitos deletérios sobre mãe e filho.

\section{REFERÊNCIAS}

1. Rennò Jr, Ribeiro, HI. Tratado de saúde mental da mulher. 1.ed. São Paulo: Atheneu, 2012. p. 89-98.

2. Camacho, R.S. et al Transtornos Psiquiátricos na gestação e no puerpério: classificação, diagnóstico e tratamento. Revista de Psiquiatria clínica. 2006;33(2):1-16, https://doi.org/10.1590/S0101-60832006000200009.

3. Cantilino, Zambaldi, Sougey, Rennó Jr. Transtornos Psiquiátricos no pós parto. Revista de Psiquiatria clínica. 2010;37(6): 278-84, https://doi.org/10.1590/S0101-60832010000600006.

4. Costa R, Pacheco A, Figueiredo B. Prevalência e preditores de sintomatologia depressiva após o parto. Ver Psiquiatria Clínica. 2007; 34(4): 167-165, https://doi.org/10.1590/S0101$\underline{60832007000400001 .}$

5. Ferreira Mas. A depressão no processo de maternidade: estudo prospectivo de mulheres da 36a semana de gravidez, 2aㅡ, e 6a semana pós-natal. [Tese de mestrado]. Porto: ICBAS; 2007

6. Santos MF, Martins FC, Pasquali L, Gorenstein C, Andrade LH, \& Zuardi AW. Escala de autoregistro de depressão pós-parto: estudo no Brasil. Escalas de avaliação clínica em psiquiatria e psicofarmacologia. São Paulo: Lemos Editorial, 2000, 97-101.

7. Beck CT. Revision of the postpartum depression inventory. J. Obstet Gynecol Neonatal Nurs. 2002; 31(4): 394-402, https://doi.org/10.1111/j.1552-6909.2002.tb00061.x.

8. Jl Cox .Edinburgh Postnatal Depression. British Journal of Psychiatry (1987), 150,782-786, https://doi.org/10.1192/bjp.150.6.782.

9. Augusto A, Kumar R, Calheiros JM, Matos E, Figueiredo E. Postnatal depression in an urban area of Portugal: comparision of chilg-bearing woman and matched controls. Psychol Med, 26 (1): 135-41; 1996, https://doi.org/10.1017/S0033291700033778 
10.Ruschi,Gustavo Enrico Cabral et al. Aspectos epidemiológicos da depressão pós-parto em amostra brasileira. Revista Psiquiatria Rio Grande do Sul. 2007;(29)3:274-80, https://doi.org/10.1590/S0101-81082007000300006.

11.Araújo FGMA, Gomes APR, França PS. Depressão no ciclo gravídico-puerperal. Comunicação. ciênc. Saúde. 2008;19(1): 51-60.

12.Da Silva Cruz, Eliane Bezerra; Simões, Gláucia Lucena; Faisal-Cury, Alexandre. Rastreamento da depressão pós-parto em mulheres atendidas pelo Programa de Saúde da Família. Revista Brasileira Ginecologia Obstetrícia.4(27): 181-8, 2005, https://doi.org/10.1590/S010072032005000400004

13.Lopez JRRA, Pedalini, R. Depressäo pós-parto: revisäo epidemiológica, diagnóstica e terapêutica. Inf. Psiquiatria. 1999;4(18):115-8.

14. Robertson, Emma et al. Antenatal risk factors for postpartum depression: a synthesis of recent literature. General hospital 2004;4(26):289-295, https://doi.org/10.1016/i.genhosppsych.2004.02.006

15.Wisner, Katherine L.; Parry, Barbara L.; Piontek, Catherine M. Postpartum depression. New England Journal of Medicine. 2002; 3(347):194-1999, https://doi.org/10.1056/NEJMcp011542.

16.Llewellyn AM, Stowe ZN, Nemeroff CB. Depression during pregnancy and the puerperium. J Clin Psychiatry 1997; 58:26-32

17.Righetti-Veltema, Marion Et al. Risk factors and predictive signs of postpartum depression. Journal of affective disorders. 1998;3(49):167-180, https://doi.org/10.1016/S01650327(97)00110-9

18.O'hara, Michael W.; Swain, Annette M. Rates and risk of postpartum depression-a metaanalysis. International review of 1996;1(8):37-54, https://doi.org/10.3109/09540269609037816.

19.Gaynes, Bradley N. et al. Perinatal depression: prevalence, screening accuracy, and screening outcomes: summary. 2005.

20.Cantilho A, Sougey EB. Escalas de triagem para depressão pós-parto. Neurobiologia. 2003; 65:75-79

21.Sadock BJ, Sadock AS, Kaplan \& Sadock, Compêndio de Psiquiatria. 9 ed. Porto Alegre: Artmed, 2007 